\title{
Passive exposure attenuates distraction during visual search
}

\author{
Bo-Yeong Won ${ }^{1}$, Joy J. Geng ${ }^{1,2}$ \\ 1.Center for Mind and Brain, University of California Davis, Davis, California \\ 2.Department of Psychology, University of California Davis, Davis, California
}

\begin{abstract}
Distractions are ubiquitous in our sensory environments. How do we keep them from capturing attention? Existing research has focused primarily on mechanisms of strategic control or statistical learning, both of which require knowledge (explicit or implicit) of what features belong to distractors before suppression occurs. Here, we test the hypothesis that task-free exposure to stimuli is sufficient to attenuate their effect as distractors later on. In three experiments, subjects were exposed to either colored or achromatic circles on "circle displays" interleaved with "target search displays". Later, new distractors were introduced into the search displays using colors from the circle displays. We consistently found that passively viewed colors produced less interference when introduced as new visual search distractors. We conclude that learning during passive exposure was due to habituation mechanisms that attenuate sensory responsivity to recurring stimuli, allowing attention to operate more efficiently to select task-relevant targets or novel stimuli.
\end{abstract}

\section{Keywords}

attention; distractor suppression; habituation; visual search

\section{INTRODUCTION}

The world is filled with distractions and the ability to ignore them is critical for goaloriented behaviors to succeed. Two main theories exist within the literature for how attention attenuates distractor processing. The first hypothesizes that distractors are suppressed through "top-down" strategic control (Arita, Carlisle, \& Woodman, 2012; Carlisle, Arita, Pardo, \& Woodman, 2011). The second hypothesizes that distractor suppression occurs through statistical learning of experienced distractors (Chetverikov, Campana, \& Kristjansson, 2017; Ferrante et al., 2018; Geyer, Muller, \& Krummenacher, 2006; Leber, Gwinn, Hong, \& O’Toole, 2016; Stilwell \& Vecera, 2019b; Vatterott \& Vecera, 2012; Wang \& Theeuwes, 2018; Won, Kosoyan, \& Geng, 2019; Zhang, Allenmark, Liesefeld, Shi, \& Müller, 2019). A core supposition of both is that distractor suppression occurs only after the observer has knowledge, explicit or implicit, of what defines a distractor within the current

Address correspondence to: Bo-Yeong Won, bywon@ucdavis.edu.

Author contributions

B.W. and J.J.G developed the study concept and design, collected data, performed the data analysis and interpretation, and wrote the manuscript. All authors approved the final version of the manuscript for submission. 
context. These theories imply that one must know what features belong to distractors before they can be suppressed. The current studies test a new hypothesis that attention to distractors may be attenuated, but not necessarily eliminated, by habituation, a non-associative or taskfree learning mechanism that reduces neural responses to stimuli based on passive exposure (Bonetti \& Turatto, 2019; Chelazzi, Marini, Pascucci, \& Turatto, 2019; Turatto, Bonetti, Pascucci, \& Chelazzi, 2018).

Habituation is characterized by the progressive decrease in an orienting response to sensory stimulation that cannot be explained by sensory adaptation or fatigue (Gover \& Abrams, 2009; Rankin et al., 2009; Sokolov, 1963; Thompson, 2009). Habituation is a form of nonassociative learning that is ubiquitous across species. It is hypothesized to serve as a precursor to other types of learning or sensory processing by filtering out stimuli that are irrelevant or unsurprising (Ramaswami, 2014). One potential importance for attention lies in its role as a 'firewall' that "triages sensory information into actionable and non-actionable categories" (Poon \& Young, 2006). In other words, habituation may reduce the amount of information that attention must adjudicate between by filtering stimuli that are predictably unimportant for behavior, leaving only more relevant or unpredicted stimuli for further processing. In natural environments where much information is stable over time, habituation can operate continuously to dampen responsivity to sensory noise and enhance attentional mechanisms to select targets and suppress specific distractors (Desimone \& Duncan, 1995).

Although habituation mechanisms are ubiquitous across animal species, its effect on visual attention has only been recently proposed (Turatto \& Pascucci, 2016). Turatto and colleagues reported habituation of oculomotor responses to recurring salient distractors (Bonetti \& Turatto, 2019; Chelazzi et al., 2019; Turatto et al., 2018; Turatto, Bonetti, \& Pascucci, 2018). The results of these studies are analogous to the earliest studies of habituation on the motor orienting reflex (Barry, 2009; Harris, 1943; Sokolov, 1963; Thompson \& Spencer, 1966) and suggest that habituation mechanisms contribute to learned distractor suppression. However, habituation was measured within the context of an active task in most of these studies, which left open the possibility that other mechanisms (e.g., associative or strategic ones) also contributed to the behavioral effects. Only one study has provided direct evidence that habituation occurs with passive exposure to salient visual stimuli (Turatto, Bonetti, Pascucci, et al., 2018). In that study, observers were asked to passively view a series of displays, some of which included a salient (i.e., luminance onset) stimulus. When the salient stimulus later became a distractor during a target discrimination task, attentional capture was attenuated compared to when the displays were not first passively viewed. The authors concluded that the passive exposure habituated the visual system to the salient stimulus, which reduced its effective saliency as a distractor during a later active task.

Two questions remained, which are essential to understanding the contribution of habituation on distractor suppression. The first methodological issue is whether the habituation effects depended on the use of identical stimulus displays during passive exposure and the active task. Because the same stimuli were used during both, it is possible that surreptitious suppression of the salient stimulus during passive viewing led to direct benefits in suppression during the active task. The second theoretical issue relates to the 
presence of residual interference from the salient distractor once the active task started. The authors attributed the cost to changes in task demands when the active task began (e.g., adjusting to new stimulus-response mappings). An alternative possibility, however, is that the residual interference from previously habituated distractors reflects the true degree of stimulus attenuation afforded by habituation mechanisms for visual attention.

In order to address these two open questions, we use a novel two-phase paradigm. During the first "training" phase, observers were passively exposed to one set of colors on "circle displays" while actively learning about the target and another set of distractors on "visual search" displays. Importantly, we used non-salient colors for all non-targets in order to reduce the possibility of active suppression that is often induced by salient bottom up signals (Bravo \& Nakayama, 1992; Koch \& Ullman, 1985; Wolfe \& Horowitz, 2017). The circle displays were also visually distinct (and retinotopically non-overlapping) with the visual search displays, rendering confusion between the two unlikely. During the second "testing" phase, a subset of the "habituated" colors from the circle displays were introduced as new visual search distractors. Critically, the new visual search distractors only resembled the circle displays in terms of the color spectrum used (and not the shapes or stimulus locations) reducing the likelihood that the two stimulus displays would be directly associated (see Figure 1). Performance on new distractor visual search trials (with the habituated colors) was contrasted against a control group that only saw achromatic circles on "circle" displays during training. We hypothesized that the new distractors would produce less interference in the group that saw the color circles due to habituation.

Our experiments collectively address the first open question of whether habituation operates independently of active suppression (i) by using non-salient passive displays that are interleaved with the active search task and have very different visual properties and (ii) by directly asking observers to attend to a non-color property of the circle displays (in Experiment 2). It also addresses the second open question regarding the source of previously observed residual interference of the habituated by holding task demands constant throughout the training and testing phases. This novel task affords measurement of the unique effect of prior exposure to colored stimuli on attentional interference by new distractors.

\section{GENERAL METHODS}

\section{Stimuli and apparatus}

Each participant sat in a sound- attenuated, dimly lit room, around $60 \mathrm{~cm}$ from the monitor. Stimuli were displayed on a 27-in. Asus LCD monitor $(2,560 \times 1,440$ pixels, $60 \mathrm{~Hz})$. Stimuli were generated using MathWorks with Psychtoolbox extensions (Brainard, 1997; Pelli, 1997). Search displays: Search displays contained three colored squares (i.e., distractors) and a gray square (i.e., target). Colors for distractors were chosen from 16 equally spaced colors that only varied in hue (CIELAB space; $\mathrm{L}^{*}=70$, center: $\mathrm{a}=0, \mathrm{~b}=0$, radius of 39 ; Table 1, Bae, Olkkonen, Allred, \& Flombaum, 2015). The gray color for the target stimulus was generated by averaging all 16 distractor colors. During training (128 trials), a gray target appeared with three fixed distractors (colors 16, 2, 4, or 8, 10, 12 counterbalanced between subjects, Table 1). 
During testing (256 trials), the same gray target appeared with one of three sets of distractors - trained (i.e., the same distractors seen during training), trained-similar $(1,3,5$ or $7,9,11)$, and new $(8,10,12$ or 16, 2, 4). Trained distractor displays appeared six times more frequently (192 trials) than the trained-similar or new distractor trials ( 32 trials each). The uneven distribution was used to maintain distractor expectations to minimize the immediate effect of active suppression on the trained-similar and new distractors. The trained-similar condition was included to add variability in the distractor sets, but we expected learned suppression from the trained distractors to generalize to the trained-similar color set because of its similarity (Won and Geng, 2018). Each square subtended $1.1^{\circ} \times 1.1^{\circ}$ of horizontal and vertical visual angle, and was located in one of four quadrants and centered $1.80^{\circ}$ of horizontal and vertical visual angle from the fixation cross. On each trial, each square was randomly assigned one number ( 1 through 4 ) drawn in white. The task was to manually report the number within the target. Auditory feedback was provided (a three "chirp" sequence lasting 300-msec for correct responses; a single high-pitched 100-msec tone followed by an additional 3-sec blank period for incorrect responses).

\section{Circle displays}

Circle displays contained four circles (diameter: $1.1^{\circ}$ ). Each circle was presented in one quadrant, and randomly jittered between $-.4^{\circ}$ and $+.4^{\circ}$ of horizontal and vertical visual angle. The placement of each circle was randomly chosen among 12 locations. The 12 possible locations were drawn from $4 \times 4$ grid $\left(8.9^{\circ} \times 8.9^{\circ}\right)$ that excluded the four most central locations (i.e., close to the fixation). The central locations were avoided because they overlapped with the visual search displays. The habituation group saw the four circles filled with four different colors chosen from 180 equally spaced colors that only varied in hue (the same parameters with those for distractor colors). In each display, each of four colors was at least 10 consecutive colors $\left(20^{\circ}\right)$ apart from each other in a color wheel that consists of 180 colors to prevent color uniformity. For the control group, circles were not filled with any colors but just drawn with a white outline Analysis

Overall accuracy was high in all experiments (Experiment 1: habituation group: 97.8\%, control group: 96.8\%; Experiment 2: habituation group: 97.5\%, control group: 96.9\%; Experiment 3: habituation group: 96.7\%, control group: 97.2\%). We therefore only use RT data from testing trials in subsequent analyses to test our hypotheses regarding the attenuation of attention to new distractors in the habituation vs. control groups. RTs were trimmed per person to exclude values within a condition greater than 3 standard deviations from the mean.

\section{EXPERIMENT 1}

Methods

Participants-Forty undergraduates from UC Davis participated for course credit (habituation group: $\mathrm{N}=20$, mean age $=21.3$ years, $\mathrm{SD}=2.5$, range $=18-27,16$ females, 0 lefthanded; control group: $\mathrm{N}=20$, mean age $=20.5$ years, $\mathrm{SD}=1.9$, range $=18-26,20$ females, 2 left-handed). The sample size of 20 was determined based on the previous study from which we adopted the experimental design (Won \& Geng, 2018). All participants had normal or 
corrected-to-normal vision and provided informed consent in accordance with NIH guidelines provided through the UCD Institutional Review Board.

Design and procedure-Each trial began with a 200-ms blank followed by 200-ms circle display, a 200-ms blank screen, a 200-ms fixation display, and then the target search display. Participants were instructed that there was no task associated with the circle displays and they could be ignored, but that the central display of squares in the search displays was always task relevant. Participants were also told they should search for the gray square in the search display and report the number inside using the keyboard as rapidly as possible without sacrificing accuracy. The search display was removed immediately after response. Offset of the search display was followed by $200-\mathrm{ms}$ of a white fixation and auditory feedback (Figure 1).

Results

First, to test the hypothesis that the habituation group should experience less interference when new distractors are introduced, we directly compared search RT interference in the habituation and control groups using a one-tailed t-test. The results were consistent with our hypothesis, showing the habituation group experienced significantly less interference than the control group from new distractors during testing, $t(38)=2.214, p=.016$, Cohen's $\mathrm{d}$ $=.700, \mathrm{BF}_{10}=3.962$. Second, we tested the hypothesis that despite attenuation, the habituation group would continue to experience some interference. To test this, we compared the mean RT in each group against zero. We found a significant effect in both groups (habituation group: $t(19)=2.443, p=.012$, Cohen's $d=.546, \mathrm{BF}_{10}=4.828$; control group: $t(19)=3.764, p<.001$, Cohen's $\mathrm{d}=.842, \mathrm{BF}_{10}=57.657$. These results are consistent with our hypotheses and demonstrate that interference from new distractors was attenuated following passive exposure, but not eliminated (Figure 2).

RT across blocks-We next examined how interference from the new distractors changed as a function of direct experience. We hypothesized that interference would decrease over time in both groups, consistent with previous findings that statistical learning of distractor features can eliminate interference entirely (Won et al., 2019; Vatterot, Mozer, \& Vecera, 2018). To test the effect of direct experience with new distractors on search interference in each group, the data from the testing phase were divided into four blocks and compared against zero. This resulted in statistically significant (non-zero) interference in blocks 1 and 2 but not in blocks 3 and 4 in the habituation group ( $1^{\text {st }}$ block: $t(19)=2.059, p=.027$, Cohen's $\mathrm{d}=.460, \mathrm{BF}_{10}=2.549 ; 2^{\text {nd }}$ block: $t(19)=2.248, p=.018$, Cohen's $\mathrm{d}=.503, \mathrm{BF}_{10}$ $=3.466 ; 3^{\text {rd }}$ block: $t(19)=.043, p=.483, \mathrm{BF}_{01}=4.165 ; 4^{\text {th }}$ block: $t(19)=.779, p=.223$, $\left.\mathrm{BF}_{01}=2.145\right)$. There was more than four times the evidence for the null than the alternative hypothesis in block 3 and two times in block 4. In contrast, all four blocks in the control group were significant, although the BFs indicate that evidence for a difference decreased over time ( $1^{\text {st }}$ block: $t(19)=3.475, p=.001$, Cohen's $\mathrm{d}=.777, \mathrm{BF}_{10}=32.646 ; 2^{\text {nd }}$ block: $t(19)=2.852, p=.005$, Cohen's $\mathrm{d}=.638, \mathrm{BF}_{10}=10.002 ; 3^{\text {rd }}$ block: $t(19)=3.098, p=.003$, Cohen's $\mathrm{d}=.693, \mathrm{BF}_{10}=15.841 ; 4^{\text {th }}$ block: $t(19)=1.905, p=.036$, Cohen's $\mathrm{d}=.426, \mathrm{BF}_{10}$ $=2.005$ ).

J Exp Psychol Gen. Author manuscript; available in PMC 2021 October 01. 
These results suggest that direct experience with the new distractors led to continued attenuation of interference from those distractors in both groups. However, because the habituation group began the testing phase with less interference by the new distractors, interference was completely eliminated after two blocks of active experience. The interference was never eliminated in the control group, but there was a steady decline in the degree of interference. This continued attenuation can be seen in the reduction of evidence in favor of the alternative hypothesis over each block, ranging from very strong evidence in block 1 to weak evidence in block 4 (Figure 3).

\section{EXPERIMENT 2}

Experiment 1 showed that passive exposure to colors in circle displays attenuated interference from new distractors in subsequent active visual search. However, it is possible that our instruction to ignore the circle displays might have encouraged participants to surreptitiously and actively suppress the circle displays, including the colors - a behavior we wished to avoid. To rule out the possibility of surreptitious suppression of the circle colors, we asked participants to count the number of circles in the circle displays. The counting task forced observers to attend to the circles but not to their colors. To preview the results, we replicated the results from Experiment 1, suggesting that the attenuation of interference in the habituation group is not due to a surreptitious "active suppression" of stimuli but rather due to the passive processing of colors.

\section{Methods}

Participants-Forty undergraduates (20 each group) from UC Davis (habituation group: $\mathrm{N}=20$, mean age $=19.8$ years, $\mathrm{SD}=1.5$, range $=18-24 ; 17$ females; 1 left-handed; control group: $\mathrm{N}=20$, mean age $=21.0$ years, $\mathrm{SD}=3.5$, range $=18-33 ; 14$ females; 2 left-handed) participated for course credit.

Design and procedure-Everything was identical to Experiment 1 except that the circle display was occasionally ( $12.3 \%$ of trials, 54 trials) followed by a number question that asked how many circles ( 3,4 or 5$)$ were present in the preceding display in the training and testing phases. The search display was identical to Experiment 1 in the remaining trials $(87.7 \%, 384$ trials) (Figure 4).

\section{Results}

Counting Accuracy-The accuracy of counting task did not differ between groups, (habituation group: 92.1\%; control group: $89.7 \%$ ), $t(38)=.825, p=.414, \mathrm{BF}_{01}=2.471$, suggesting a similar degree of attention paid to the colored and achromatic circles.

Mean RT-Similar to Experiment 1, we first tested RT interference between groups and found a significant difference, $t(38)=2.011, p=.026$, Cohen's $\mathrm{d}=.636, \mathrm{BF}_{10}=2.838$ (Figure 5). In replication of Experiment 1, the attenuation of interference in the habituation group was stronger than in the control group. To quantify this expected null difference, we compared the magnitude of interference between experiments using Bayesian ANOVA (JASP version 0.11.1). We specified the alternative model with RT interference as the 
dependent variable, with group (control and habituation) and experiment (exp1 and exp2) and their interaction as fixed factors. The null model only included the factor of group (control, habituation), which was significant in both experiments (see above). The default prior for fixed effects $(.5)$ was used. The results found no evidence for a betweenexperiments interaction, $\left(F<1, p=.574, \mathrm{BF}_{01}=12.167\right)$. Moreover, the $\mathrm{BF}$ indicates there is more than twelve times the evidence for the null model than the alternative, suggesting that the effect of group on the magnitude of new distractor interference did not differ across experiments. This result demonstrates that the attenuation of interference was equivalent between experiments, rendering the possibility that the results from Experiment 1 were due to surreptitious active suppression of colored circles in the circle displays unlikely.

Next, we compared RT interference in each group against zero to test the hypothesis that there would be residual interference in the habituation condition. Consistent with this, we again found strong evidence for significant interference by new distractors in both groups (habituation group: $t(19)=3.180, p=.002$, Cohen's $d=.711, \mathrm{BF}_{10}=18.494$; control group: $t(19)=4.829 p<.001$, Cohen's d $\left.=1.080 ., B_{10}=484.879\right)$.

RT across blocks-We next examined RT interference in each group across blocks in order to test if distractor attenuation increases with experience of the new distractors. The habituation group experienced significant interference in blocks 1 and 2, but examination of the BFs suggests caution in interpretation since evidence for either the alternative or null hypothesis in each block was weak ( $1^{\text {st }}$ block: $t(19)=1.709, p=.052$, Cohen's $\mathrm{d}=.382$, $\mathrm{BF}_{10}=1.496 ; 2^{\text {nd }}$ block: $t(19)=2.646, p=.008$, Cohen's $\mathrm{d}=.592, \mathrm{BF}_{10}=6.894 ; 3^{\text {rd }}$ block: $t(19)=1.650, p=.058$, Cohen's $\mathrm{d}=.369, \mathrm{BF}_{10}=1.374 ; 4^{\text {th }}$ block: $t(19)=1.612, p=.062$, Cohen's $\mathrm{d}=.360, \mathrm{BF}_{10}=1.301$ ). Altogether, the results suggest that there was little to no interference across all four blocks. The control group showed significant interference in blocks $1-3\left(1^{\text {st }}\right.$ block: $t(19)=3.745, p<.001$, Cohen's $\mathrm{d}=.837, \mathrm{BF}_{10}=55.495 ; 2^{\text {nd }}$ block: $t(19)=2.662, p=.008$, Cohen's d $=.595, \mathrm{BF}_{10}=7.095 ; 3^{\text {rd }}$ block: $t(19)=3.626, p<.001$, Cohen's $\mathrm{d}=.811, \mathrm{BF}_{10}=43.953 ; 4^{\text {th }}$ block: $t(19)=1.223, p=.118$, Cohen's $\mathrm{d}=.274, \mathrm{BF}_{01}$ $=1.288$ ). The pattern of results again suggests that RT interference decreased over time, consistent with the notion that learned distractor suppression operates over and above that of habituation alone.

\section{EXPERIMENT 3}

In the previous two experiments, we found that passive exposure to colors in the circle displays attenuated, but did not eliminate, interference from new distractors in later visual search displays. However, because the colors of circle displays were randomly chosen from the entire range of the color wheel, only a subset of circle displays contained colors that matched the new distractors; this might have resulted in relatively weak habituation (therefore leaving residual interference). If more frequent and specific exposure to colors that match new distractors during training leads to stronger habituation, then using a narrower set of circle colors should lead to greater suppression of new distractors later on during the testing phase. Additionally, to confirm that habituation only occurs for the seen colors and does not generalize from very different colors (i.e., preserves stimulus specificity; Rankin et al., 2009), we added colors to the control group circle displays that matched the 
trained distractors (Figure 7). We hypothesized that the habituation group would experience less interference from new distractors than the control group, in replication of Experiments 1 and 2 .

\section{Methods}

Participants-Forty undergraduates (20 each group) from UC Davis (habituation group: $\mathrm{N}=20$, mean age $=21.7$ years, $\mathrm{SD}=2.2$, range $=19-26 ; 14$ females; 0 left-handed; control group: $\mathrm{N}=20$, mean age $=21.5$ years, $\mathrm{SD}=2.9$, range $=18-29 ; 9$ females; 1 left-handed) participated for course credit.

Design and procedure-Everything was identical to Experiment 1 except that the colors of circles in the circle displays were chosen from a limited range (Figure 7). Each of four colors in the circle display was at least 8 consecutive colors $\left(16^{\circ}\right)$ apart from each other in a color wheel that consists of 180 colors. During the training phase, the habituation group only saw circle displays with colors from a restricted range opposite to trained visual search distractors. The colors seen by the habituation group overlapped with future new distractors. The control group saw circle displays with colors from a restricted range that overlapped with the trained and trained-similar distractor sets. As in the previous experiments, there was no difference in performance between the trained and trained-similar distractors and therefore the data were collapsed. The distractors in visual search displays were identical to those in previous experiments.

\section{Results}

Mean RT-Similar to previous experiments, we first compared RT interference directly between groups and again found less interference from new distractors in the habituation group compared to the control group, $t(38)=3.007, p=.002$, Cohen's $\mathrm{d}=.951, \mathrm{BF}_{10}=$ 17.909 (Figure 8). Importantly, we again replicate the finding that there was residual interference in the habituation group: both groups showed significant interference from new distractors compared to zero (habituation group: $t(19)=3.154, p=.003$, Cohen's $\mathrm{d}=.705$, $\mathrm{BF}_{10}=17.615$; control group: $t(19)=6.176, p<.001$, Cohen's $\mathrm{d}=1.381, \mathrm{BF}_{10}=6795.112$ ).

In order to quantify the similarity of the results with Experiments 1, we compared the magnitude of attenuation to new distractors between experiments by using a Bayesian ANOVA with factors experiment (exp1, exp3) and group (control, habituation). The interaction was not significant, suggesting a comparable magnitude of attenuation in the habituation group compared to the control group between experiments, $F<1, p=.693, \mathrm{BF}_{01}$ $=11.701$. The BF, which compared the full model against a null model that included only the factor of group, indicates more than eleven times the evidence for the null model. This suggests that the effect of group on the magnitude of new distractor interference did not differ across experiments. A similar result was found when comparing interference between Experiments 2 and $3\left(F<1, p=.796, \mathrm{BF}_{01}=12.970\right)$.

The fact that there is residual interference following habituation even when habituation training was increased, and that the size of interference did not differ between experiments, 
suggests that habituation mechanisms in this task may afford some attenuation but not complete elimination of interference from new distractors.

RT across blocks-As in the previous experiments, we next examined whether the residual interference in the habituation group to new distractors decreased with active experience. Comparison of RT interference in each group against zero resulted in significant effects only in first two blocks in the habituation group ( $1^{\text {st }}$ block: $t(19)=2.034, p=.028$, Cohen's d $=.455, \mathrm{BF}_{10}=2.451 ; 2^{\text {nd }}$ block: $t(19)=2.699, p=.007$, Cohen's d $=.604, \mathrm{BF}_{10}$ $=7.577 ; 3^{\text {rd }}$ block: $t(19)=1.436, p=.084$, Cohen's $d=.321, \mathrm{BF}_{10}=1.022 ; 4^{\text {th }}$ block: $t(19)$ $=.940, p=.179$, Cohen's $\mathrm{d}=.210, \mathrm{BF}_{01}=1.800$ ). This is consistent with the notion that direct experience with the new colored stimuli as active distractors led to additional suppression. In contrast, interference was significant in all blocks in the control group, although the strength of evidence for a difference also decreased over blocks ( $1^{\text {st }}$ block: $t(19)$ $=4.542, p<.001$, Cohen's d $=1.016, \mathrm{BF}_{10}=272.886 ; 2^{\text {nd }}$ block: $t(19)=2.985, p=.004$, Cohen's d $=.667, \mathrm{BF}_{10}=12.792 ; 3^{\text {rd }}$ block: $t(19)=4.193, p<.001$, Cohen's $\mathrm{d}=.938, \mathrm{BF}_{10}$ $=135.570 ; 4^{\text {th }}$ block: $t(19)=2.925, p=.004$, Cohen's $\left.d=.654, \mathrm{BF}_{10}=11.452\right)$. This pattern converges with Experiments 1 and 2, suggesting that passive exposure to the circle displays resulted in attenuated distractor interference, but that the elimination of interference only occurred with continued exposure to the colors as active distractors within the visual search task.

\section{DISCUSSION}

The primary question of interest addressed in these studies was whether passive exposure to circle displays containing non-salient colored stimuli would attenuate interference by those colors when they later appeared as distractors in visual search. The circle displays were constructed to be dissimilar from visual search displays by using objects of different shapes and presented in different configurations and at different visual eccentricities. The colors were also heterogenous and non-salient. The purpose of using these displays was to minimize the likelihood that observers would actively suppress the stimuli (e.g., by confusing them with distractors or in response to automatic bottom-up capture) and prevent retinotopic sensory adaptation. In all three experiments, we found positive evidence that passive exposure to colored stimuli reduced, but did not fully eliminate, behavioral interference when they later appeared as distractors. This suggests that habituation mechanisms during passive exposure reduced sensory responsivity and thus attentional priority to those colors when they became task-relevant distractors. Our results are novel and go beyond previous studies in several ways.

First, we introduce a novel paradigm and find consistent evidence that attention is attenuated to non-salient colored distractors that were previously seen in a task-free context. Previous studies of habituation on visual attention used salient non-targets defined by luminance and the current data extend those findings to a new feature domain. Thus, habituation appears to operate on non-salient stimuli seen in task-irrelevant contexts, suggesting that it might play an important ongoing role in natural vision where most stimuli tend to be non-salient, stable over space and time, and maintain their status as behaviorally irrelevant (e.g., imagine in an auditorium, the seating, carpet, and walls). Habituation might operate rapidly on those 
stimuli and by doing so, "triage" the vast majority of visual information from continued processing, allowing selective attention to operate on a smaller subset of plausibly important information.

Second, our results shed new light on interpretation of persistent distractor costs following habituation. All three studies found (approximately 20-msec) of residual interference following habituation, which suggests that habituation following passive exposure attenuates but does not completely eliminate attentive processing of perceptual stimuli. Similar findings have been reported in the visual (Turatto et al., 2018) as well as auditory domains (Bell, Roer, Dentale, \& Buchner, 2012). For example, Turatto et al. (2018) observed an initial RT cost that lasted for up to 10 trials following passive exposure to salient distractors. However, because their task required a change in performance demands between the period of passive exposure (no response required) and active discrimination (task response required), they attributed the remaining cost to changes in stimulus-response requirements. In our study, it was unnecessary to implement new stimulus-response mappings since the only thing that changed was the appearance of distractor colors. The fact that a performance cost remained argues that habituation following passive exposure in our training task did not completely wipe out distraction by new distractor stimuli but did attenuate priority compared to the same stimuli without prior exposure. Interference was only resolved completely after the testing phase began, which included active experience with the new distractors as well as continued exposure to the circle displays. This indicates that while habituation can occur during both passive and active tasks, the effect of habituation following passive exposure may be either weaker than under active tasks or that maximum suppression relies on the contribution of other mechanisms engaged during active tasks (Geng, Won, \& Carlisle, 2019).

Third, we introduced an active dual-task during the circle displays in Experiment 2 to test if habituation to color would occur even when subjects attend to the number of circles in the display. The circle counting task ensured that subjects attended actively to the display as a whole, which precludes the ability to suppress the circle stimuli, but also left the color feature dimension of each circle irrelevant. The fact that we continued to see a similar magnitude of distractor attenuation for the habituated colors between Experiment 1 and Experiment 2 suggests that it is the task-irrelevance of the colors that led to habituation, not that they were viewed during a passive exposure in Experiment 1. The results also suggest that irrelevant features in one context might continue to be tagged as irrelevant in the next unless there is evidence to the contrary.

Finally, our results demonstrate that habituation was specific to the exposed stimulus colors, which is predicted by models of habituation (Thompson, 2009). In Experiment 3, the habituated color stimuli on circle displays during training were limited to a specific range (e.g., bluish) and kept separate from the trained distractors (e.g., reddish) in the habituation group. The control group also saw colored habituation displays but the colors were in the same range as the trained distractors. We found that attention to new distractors were attenuated only in the habituation group that saw passive circle display colors that overlapped with new distractors. However, the fact that we also observed habituation effects in the first two studies with variable colors, suggests that habituation might flexibly occur at 
different levels of sensory processing depending on the statistics of exposure - for example, occurring at the level of a feature dimension (Müller, Heller, \& Ziegler, 1995) or a specific stimulus. Such flexibility has been observed in statistical learning paradigms of distractor suppression (Stilwell \& Vecera, 2019a; Vatterott, Mozer, \& Vecera, 2018; Vatterott \& Vecera, 2012; Won et al., 2019). Interestingly, however, the attenuation of new distractors was not stronger in Experiment 3 than in the Experiment 1, suggesting that simply seeing more specific habituation displays did not immediately increase suppression. However, it is possible that longer or more specific habituation training could have led to stronger suppression and this is a question of active investigation.

The current work provides clear evidence of a role for passive exposure, presumed to be supported by habituation mechanisms, in efficient attentional processing (Bonetti \& Turatto, 2019; Turatto, Bonetti, \& Pascucci, 2018; Turatto, Bonetti, Pascucci, et al., 2018; Turatto \& Pascucci, 2016). Habituation may serve as an early filter that attenuates processing of previously encountered sensory information that did not elicit attentive processing or a behavioral response (Gover \& Abrams, 2009; Poon \& Young, 2006; Rankin et al., 2009) Yamaguchi, 2004). This characterization relates to recent models of predictive coding (Auksztulewicz, 2017; Friston, 2012; Parr, 2018) - both theorize that neurons encode a model of the expected relevance of stimuli. The relationship between habituation and predictive coding has yet to be explored, but will be important for understanding how passive exposure improves attentional selection. In sum, our data suggest that habituation aids attentive processing by "triaging" sensory information and providing a less crowded "stage" on which more cognitively intensive attentional mechanisms may operate to adjudicate between remaining competing stimuli.

\section{Acknowledgements}

The work was supported by NSF BCS-201502778 and NIH-RO1-MH113855-01 to JJG.

\section{References}

Arita JT, Carlisle NB, \& Woodman GF (2012). Templates for rejection: configuring attention to ignore task-irrelevant features. J Exp Psychol Hum Percept Perform, 38(3), 580-584. doi:10.1037/ a0027885 [PubMed: 22468723]

Barry RJ (2009). Habituation of the orienting reflex and the development of Preliminary Process Theory. Neurobiol Learn Mem, 92(2), 235-242. doi:10.1016/j.nlm.2008.07.007 [PubMed: 18675927]

Bell R, Roer JP, Dentale S, \& Buchner A (2012). Habituation of the irrelevant sound effect: evidence for an attentional theory of short-term memory disruption. J Exp Psychol Learn Mem Cogn, 38(6), 1542-1557. doi:10.1037/a0028459 [PubMed: 22612161]

Bonetti F, \& Turatto M (2019). Habituation of oculomotor capture by sudden onsets: Stimulus specificity, spontaneous recovery and dishabituation. J Exp Psychol Hum Percept Perform, 45(2), 264-284. doi:10.1037/xhp0000605 [PubMed: 30570321]

Carlisle NB, Arita JT, Pardo D, \& Woodman GF (2011). Attentional templates in visual working memory. J Neurosci, 31(25), 9315-9322. doi:10.1523/JNEUROSCI.1097-11.2011 [PubMed: 21697381]

Chelazzi L, Marini F, Pascucci D, \& Turatto M (2019). Getting Rid of Visual Distractors: The Why, When, How and Where. Current Opinion in Psychology. doi:10.1016/j.copsyc.2019.02.004 
Chetverikov A, Campana G, \& Kristjansson A (2017). Representing color ensembles. Psychological Science.

Desimone R, \& Duncan J (1995). Neural mechanisms of selective attention. Annual Review of Neuroscience, 18, 193-222.

Ferrante O, Patacca A, Di Caro V, Della Libera C, Santandrea E, \& Chelazzi L (2018). Altering spatial priority maps via statistical learning of target selection and distractor filtering. Cortex, 102, 67-95. doi:10.1016/j.cortex.2017.09.027 [PubMed: 29096874]

Geng JJ, Won B-Y, \& Carlisle NB (2019). Distractor ignoring: strategies, learning, and passive filtering. Current Directions in Psychological Science.

Geyer T, Muller HJ, \& Krummenacher J (2006). Cross-trial priming in visual search for singleton conjunction targets: Role of repeated target and distractor features. Perception \& Psychophysics, 68(5), 736-749. [PubMed: 17076342]

Gover TD, \& Abrams TW (2009). Insights into a molecular switch that gates sensory neuron synapses during habituation in Aplysia. Neurobiol Learn Mem, 92(2), 155-165. doi:10.1016/ j.nlm.2009.03.006 [PubMed: 19345275]

Harris JD (1943). Habituatory response decrement in the intact organism. Psychological Bulletin, 40(6), 385-422.

Leber AB, Gwinn RE, Hong Y, \& O’Toole RJ (2016). Implicitly learned suppression of irrelevant spatial locations. Psychon Bull Rev, 23(6), 1873-1881. doi:10.3758/s13423-016-1065-y [PubMed: 27225635]

Poon CS, \& Young DL (2006). Nonassociative learning as gated neural integrator and differentiator in stimulus-response pathways. Behav Brain Funct, 2, 29. doi:10.1186/1744-9081-2-29 [PubMed: 16893471]

Ramaswami M (2014). Network plasticity in adaptive filtering and behavioral habituation. Neuron, 82(6), 1216-1229. doi:10.1016/j.neuron.2014.04.035 [PubMed: 24945768]

Rankin CH, Abrams T, Barry RJ, Bhatnagar S, Clayton DF, Colombo J,... Thompson RF (2009). Habituation revisited: an updated and revised description of the behavioral characteristics of habituation. Neurobiol Learn Mem, 92(2), 135-138. doi:10.1016/j.nlm.2008.09.012 [PubMed: 18854219]

Sokolov EN (1963). Perception and the Conditioned Reflex. Oxford: Pergamon Press.

Stilwell BT, \& Vecera SP (2019a). Cued distractor rejection disrupts learned distractor rejection. Visual Cognition, 1-16. doi:10.1080/13506285.2018.1564808

Stilwell BT, \& Vecera SP (2019b). Learned and cued distractor rejection for multiple features in visual search. Atten Percept Psychophys, 81(2), 359-376. doi:10.3758/s13414-018-1622-8 [PubMed: 30421366]

Thompson RF (2009). Habituation: a history. Neurobiol Learn Mem, 92(2), 127-134. doi:10.1016/ j.nlm.2008.07.011 [PubMed: 18703156]

Thompson RF, \& Spencer WA (1966). Habituation: A model phenomenon for the study of neuronal substrates of behavior. Psychological Review, 73(1), 16-43. [PubMed: 5324565]

Turatto M, Bonetti F, \& Pascucci D (2018). Filtering visual onsets via habituation: A context-specific long-term memory of irrelevant stimuli. Psychon Bull Rev, 25(3), 1028-1034. doi:10.3758/ s13423-017-1320-x [PubMed: 28547537]

Turatto M, Bonetti F, Pascucci D, \& Chelazzi L (2018). Desensitizing the attention system to distraction while idling: A new latent learning phenomenon in the visual attention domain. Journal of Experimental Psychology: General, 147(12), 1827-1850. doi:10.1037/xge0000503 [PubMed: 30359073]

Turatto M, \& Pascucci D (2016). Short-term and long-term plasticity in the visual-attention system: Evidence from habituation of attentional capture. Neurobiol Learn Mem, 130, 159-169. doi:10.1016/j.nlm.2016.02.010 [PubMed: 26927305]

Vatterott DB, Mozer MC, \& Vecera SP (2018). Rejecting salient distractors: Generalization from experience. Atten Percept Psychophys, 80(2), 485-499. doi:10.3758/s13414-017-1465-8 [PubMed: 29230673]

Vatterott DB, \& Vecera SP (2012). Experience-dependent attentional tuning of distractor rejection. Psychon Bull Rev, 19(5), 871-878. doi:10.3758/s13423-012-0280-4 [PubMed: 22696250] 
Wang B, \& Theeuwes J (2018). How to inhibit a distractor location? Statistical learning versus active, top-down suppression. Atten Percept Psychophys, 80(4), 860-870. doi:10.3758/s13414-018-1493z [PubMed: 29476331]

Won B-Y, Kosoyan M, \& Geng JJ (2019). Evidence for second-order singleton suppression based on probabilistic expectations. J Exp Psychol Hum Percept Perform, 45(1), 125-138. doi:10.1037/ xhp0000594 [PubMed: 30596437]

Zhang B, Allenmark F, Liesefeld HR, Shi Z, \& Müller HJ (2019). Probability cueing of singletondistractor locations in visual search: Priority-map- versus dimension-based inhibition? Journal of Experimental Psychology: Human Perception and Performance, 45(9), 1146-1163. doi:10.1037/ xhp0000652 [PubMed: 31144860] 


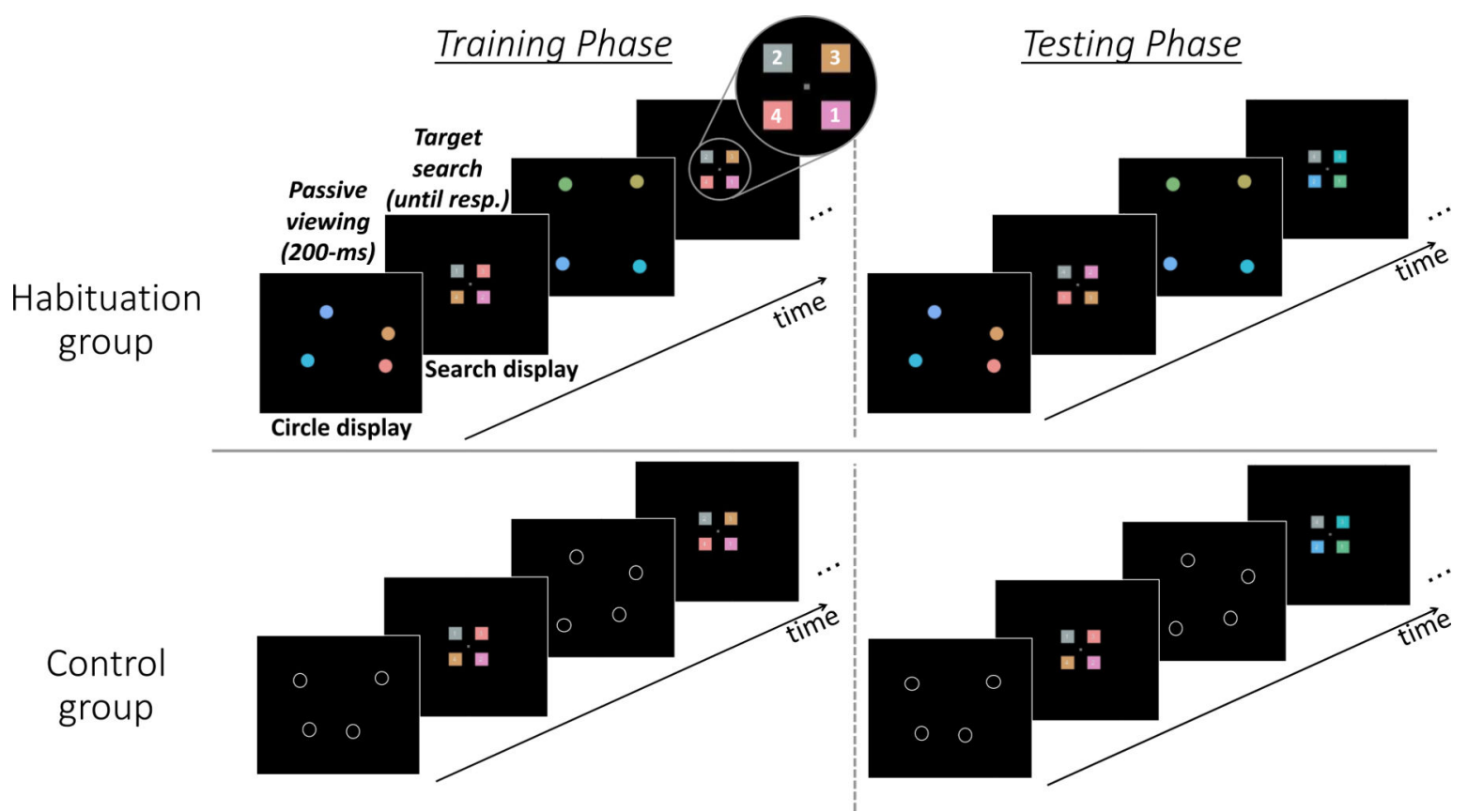

Figure 1.

Illustration of trials from Experiment 1. Participants were asked to ignore circle displays but search for a gray square (target) among colored squares (distractors) on visual search displays. In the habituation group, the circle displays had colored circles, and in the control group, the circle displays had unfilled circles. During training, the three colored distractors were always the same (trained). During testing, a new set of colored distractors was randomly interleaved with trained distractors (see text). 


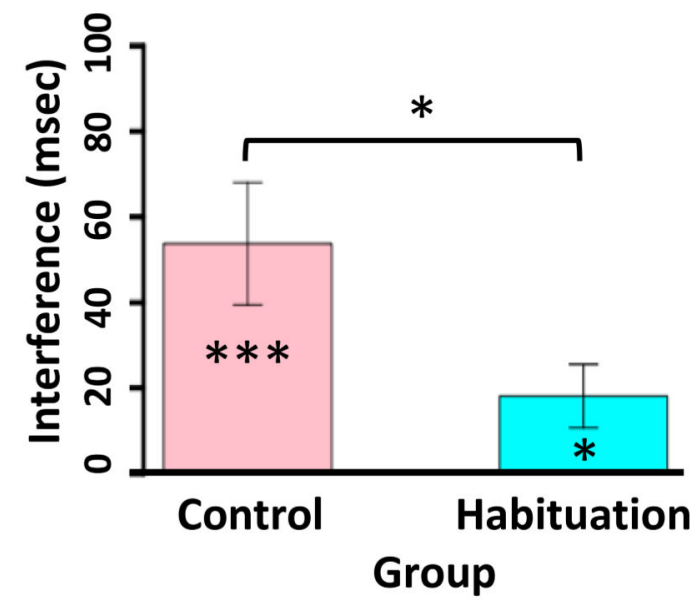

A

Figure 2.

Results from Experiment 1. A. Search interference (i.e., search RT to new distractors minus search RT to trained distractors) in the habituation and control groups. Greater distractor interference was found by new distractors in the control than habituation group, but both groups experienced significant interference. $B$. Search RT to trained and new distractor visual search trials in the control and habituation groups. Error bars show \pm betweensubjects standard error of the mean. * indicates $p<.05 ; * *$ indicates $p<.01 ; * * *$ indicates $p$ $<.001$. 


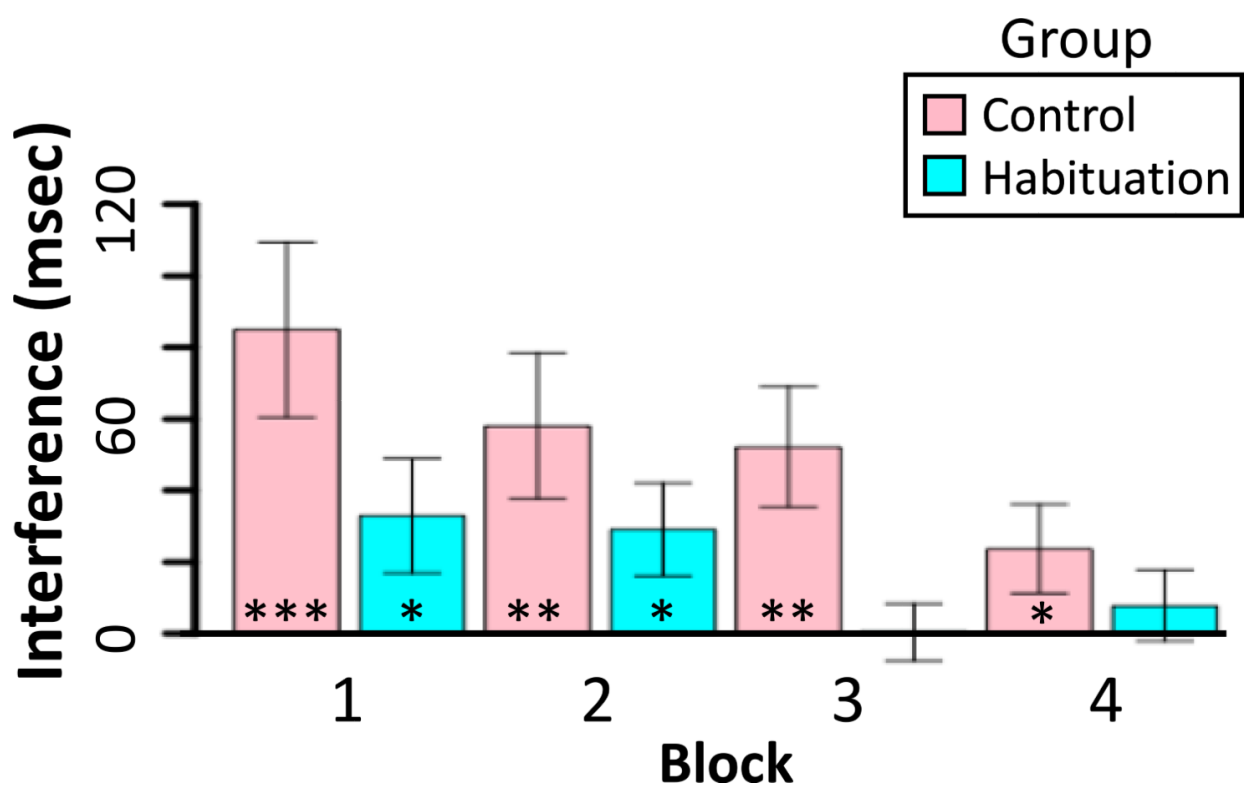

Figure 3.

Experiment 1 search RT interference (RT new minus RT trained) across four blocks in the two groups. Error bars show \pm between-subjects standard error of the mean. * indicates $p$ $<.05$; ** indicates $p<.01 ; * * *$ indicates $p<.001$. 


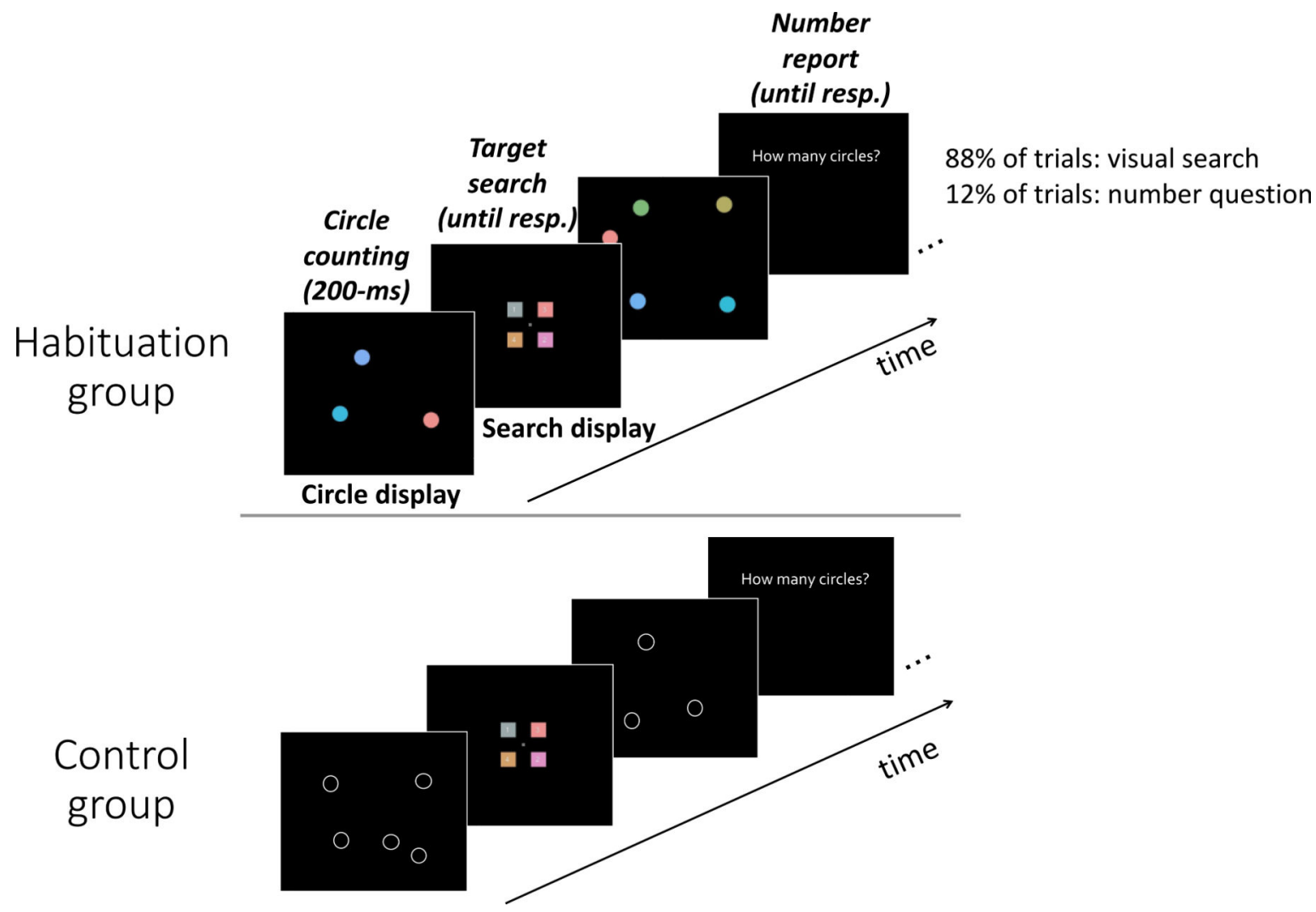

Figure 4.

An example of a two-trial sequence from Experiment 2. The first trial was composed of a circle display followed by a search display (identical to Experiment 1). On the second trial, the circle display was followed by a question regarding the number of circles on the previous display. This task ensured attention to the circle display as a whole without attention specifically to the color of the stimuli. Because the number questions occurred randomly, participants had to count circles on every circle display to perform well. Top: example trials from the habituation group; bottom: example trials from the control group. 


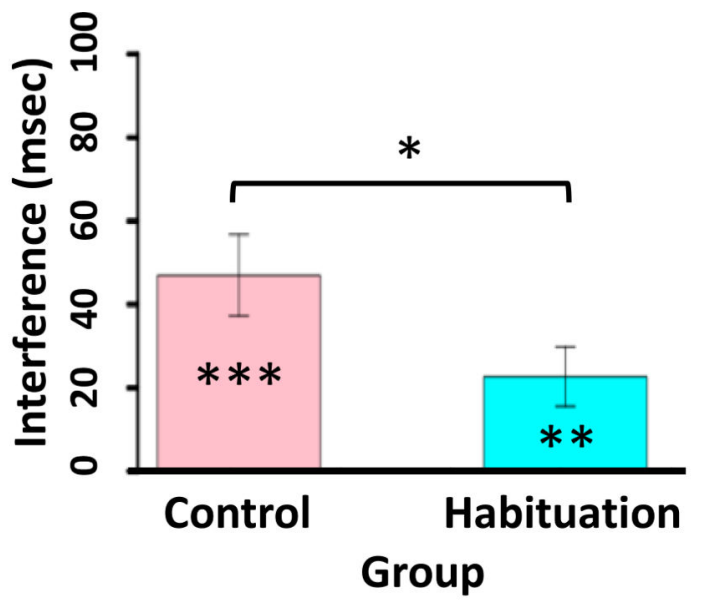

A

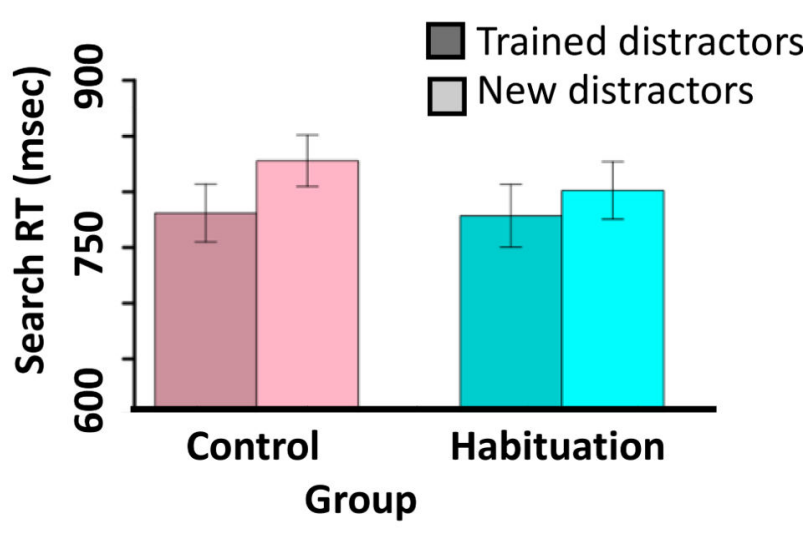

B

Figure 5.

Results from Experiment 2. A. Search interference (RT difference between trained and new distractors) in Experiment 2. B. Search RT to trained and new distractor visual search trials in the control and habituation groups. Error bars show \pm between-subjects standard error of the mean. * indicates $p<.05 ; * *$ indicates $p<.01 ; * * *$ indicates $p<.001$. 


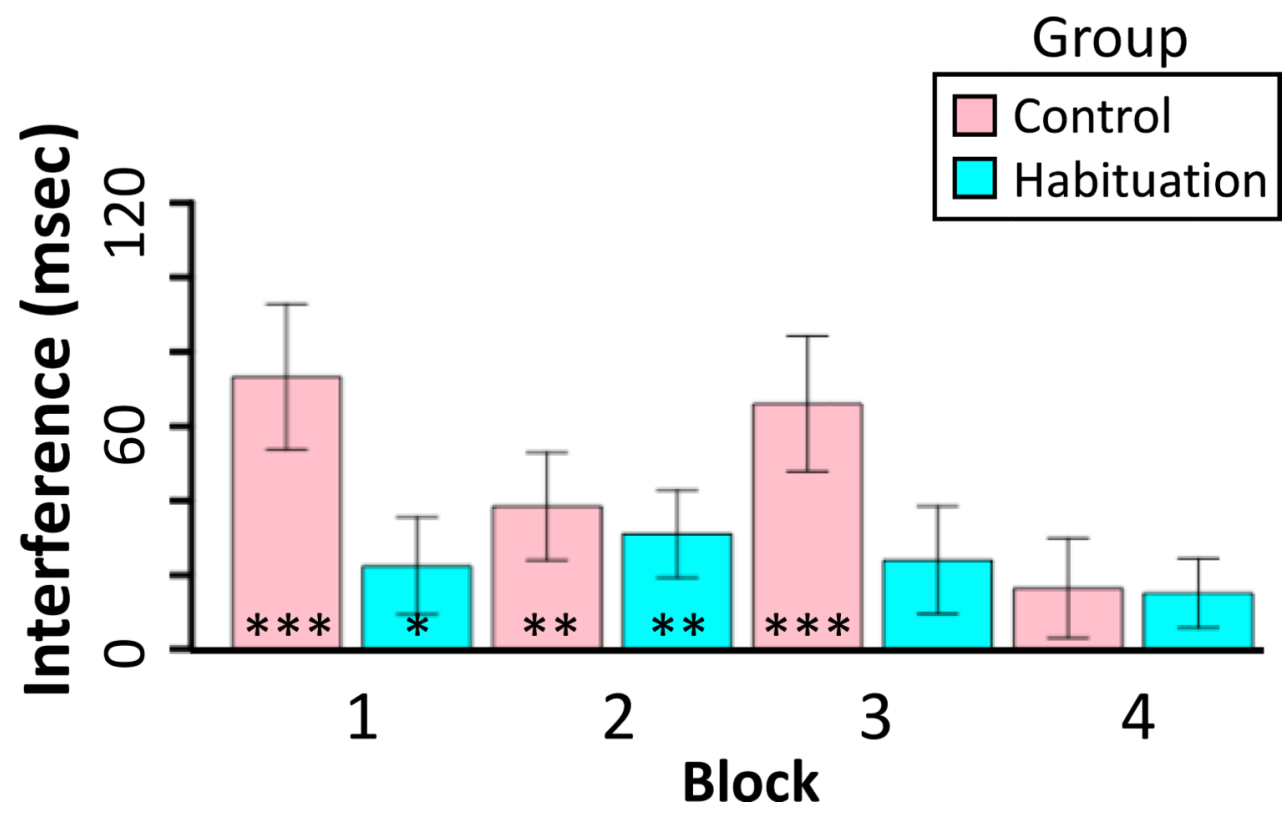

Figure 6.

Experiment 2 search interference across four blocks. Error bars show \pm between-subjects standard error of the mean. * indicates $p<.05$; ** indicates $p<.01$; *** indicates $p<.001$. 

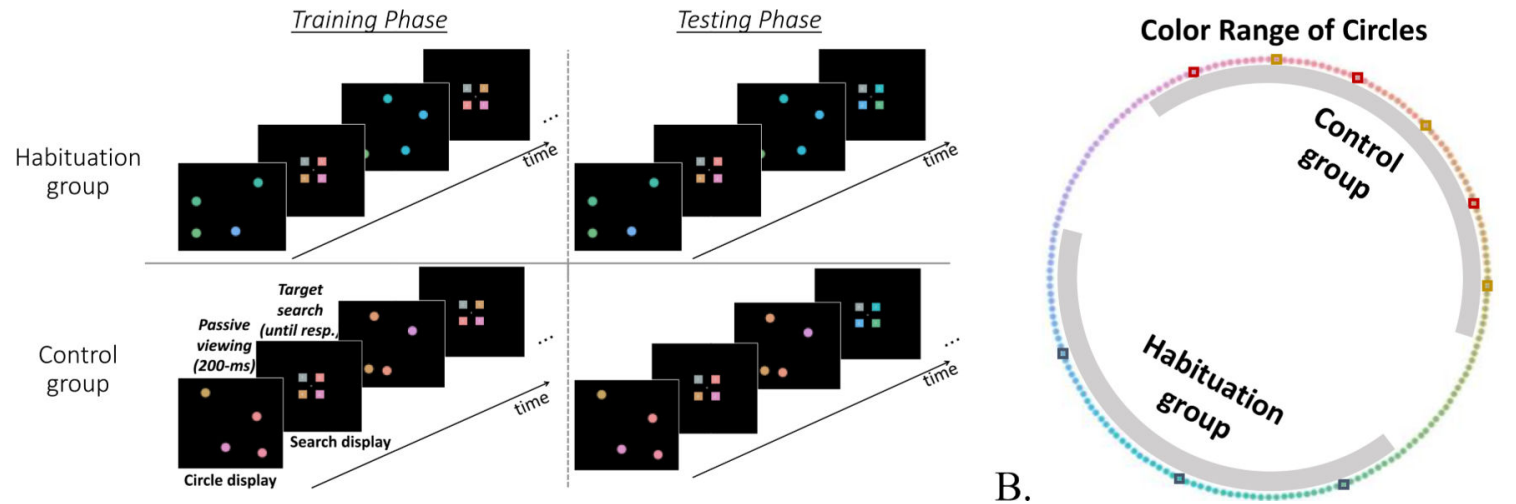

Figure 7.

Experiment 3 trial procedure. A. An example of a two-trial sequence illustrating the circle displays for both groups. B. The color wheel used in three experiments. The gray band indicates the range of colors used for the circle displays in the habituation and control groups; dark gray squares indicate new distractor colors; red squares indicate trained distractor colors; orange squares indicates trained-similar distractor colors. 


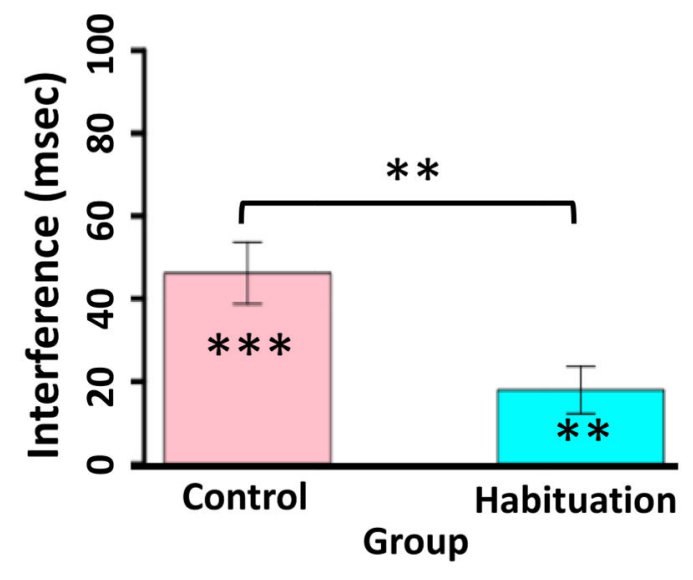

A B

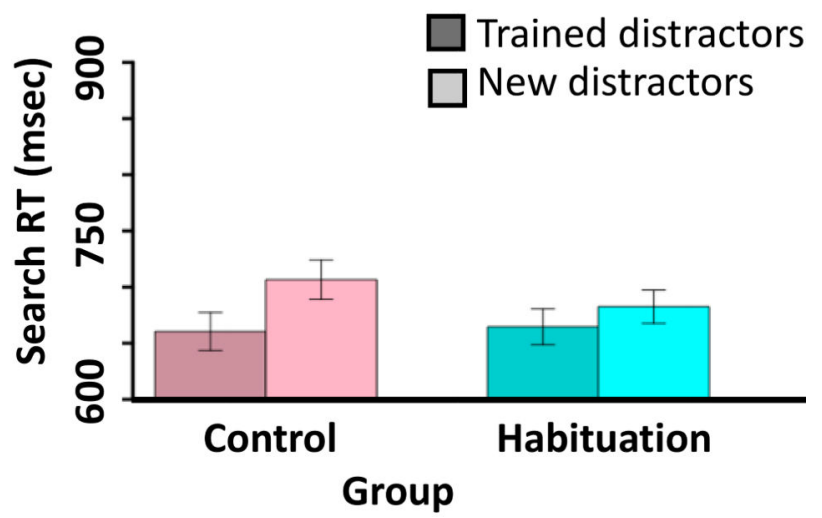

Figure 8.

Results from Experiment 3. A. Search interference (RT difference between new distractors and trained distractors) in the two groups. $B$. Search RT to trained and new distractor visual search trials in the control and habituation groups. Error bars show \pm between-subjects standard error of the mean. * indicates $p<.05$; ** indicates $p<.01$; *** indicates $p<.001$. 


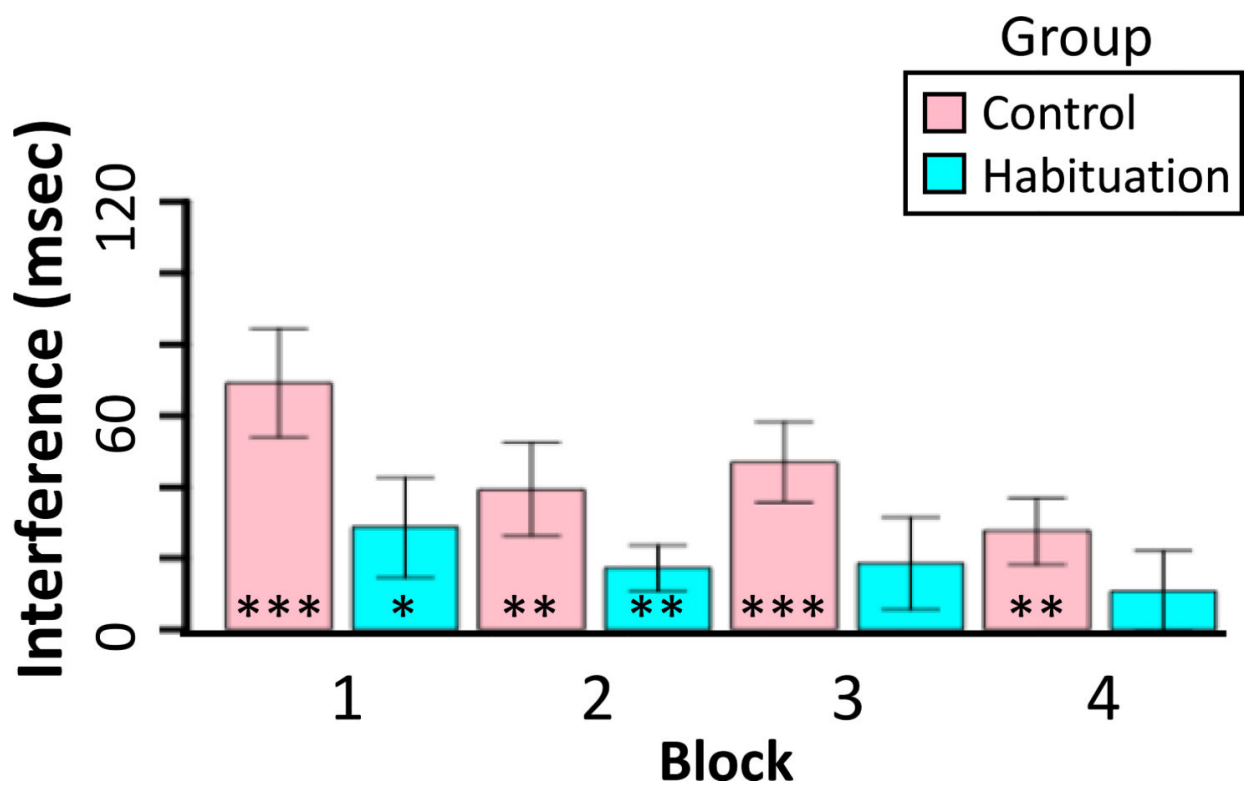

Figure 9.

Search interference across four blocks in Experiment 3. Error bars show \pm between-subjects standard error of the mean. * indicates $p<.05$; ** indicates $p<.01$; *** indicates $p<.001$. 
Table 1.

롤

distractor color coordinates in CIELAB color space

\begin{tabular}{|l|c|c|c|c|c|c|c|c|c|c|c|c|c|c|c|c|}
\hline & $\mathbf{1}$ & $\mathbf{2}$ & $\mathbf{3}$ & $\mathbf{4}$ & $\mathbf{5}$ & $\mathbf{6}$ & $\mathbf{7}$ & $\mathbf{8}$ & $\mathbf{9}$ & $\mathbf{1 0}$ & $\mathbf{1 1}$ & $\mathbf{1 2}$ & $\mathbf{1 3}$ & $\mathbf{1 4}$ & $\mathbf{1 5}$ & $\mathbf{1 6}$ \\
\hline $\mathbf{a}$ & 39 & 36 & 28 & 15 & 0 & -15 & -28 & -36 & -39 & -36 & -28 & -15 & 0 & 15 & 28 & 36 \\
\hline $\mathbf{b}$ & 0 & 15 & 28 & 36 & 39 & 36 & 28 & 15 & 0 & -15 & -28 & -36 & -39 & -36 & -28 & -15 \\
\hline
\end{tabular}

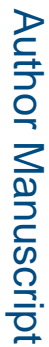

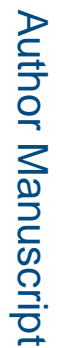

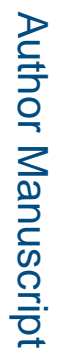

J Exp Psychol Gen. Author manuscript; available in PMC 2021 October 01. 